\title{
Helium spectrum in the Cosmic Rays measured by the DAMPE detector
}

\author{
Margherita Di Santo ${ }^{* 1,2}$,Valentina Gallo ${ }^{3}$, Peng-Xiong Ma ${ }^{4,5}$, Rui Qiao ${ }^{6}$, Yi-Feng \\ Wei $^{7,8}$, on behalf of the DAMPE Collaboration \\ ${ }^{1}$ Dipartimento di Matematica e Fisica E. De Giorgi, Università del Salento, I-73100, Lecce, Italy \\ ${ }^{2}$ Istituto Nazionale di Fisica Nucleare (INFN) - Sezione di Lecce, I-73100, Lecce, Italy \\ ${ }^{3}$ Department of Nuclear and Particle Physics, University of Geneva, CH-1211, Switzerland \\ ${ }^{4}$ Key Laboratory of Dark Matter and Space Astronomy, Purple Mountain Observatory, Chinese \\ Academy of Sciences, Nanjing 210034, China \\ ${ }^{5}$ School of Astronomy and Space Science, University of Science and Technology of China, Hefei \\ 230026, China \\ ${ }^{6}$ Institute of High Energy Physics, Chinese Academy of Sciences, Yuquan Road 19B, Beijing \\ 100049, China \\ ${ }^{7}$ State Key Laboratory of Particle Detection and Electronics, University of Science and \\ Technology of China, Hefei 230026, China \\ ${ }^{8}$ Department of Modern Physics, University of Science and Technology of China, Hefei 230026, \\ China \\ E-mail: margherita.disanto@le.infn.it
}

DAMPE (DArk Matter Particle Explorer) is a space mission promoted by the Chinese Academy of Sciences (CAS), in collaboration with Universities and Institutes from China, Italy and Switzerland. The satellite was launched on December 17th, 2015, and since then DAMPE has been stably collecting data. The DAMPE detector is composed by four sub-detectors: a Plastic Scintillator Detector (PSD), a Silicon Tungsten tracKer-converter (STK), a BGO calorimeter (BGO) and a NeUtron Detector (NUD). Two layers of PSD guarantee full detection efficiency and allow the measurement of the Cosmic Ray charge, up to Iron. Thanks to the STK, the trajectory of the incoming particles is reconstructed with a spatial resolution better than $70 \mu \mathrm{m}$ within $60^{\circ}$ of incidence angle. Furthermore, the BGO calorimeter has a depth of 32 radiation lengths $(\sim 1.6$ nuclear interaction lengths) and allows the estimation of Cosmic Ray energy with a resolution better than $40 \%$ at $800 \mathrm{GeV}$. Exploiting these features, DAMPE studies the Cosmic Ray flux with unprecedented resolution in an energy range from few tens of $\mathrm{GeV}$ up to hundreds of $\mathrm{TeV}$ and establishes a bridge between direct and indirect measurements. This work is focused on the Helium analysis and preliminary results on the flux measured up to $\sim 5 \mathrm{TeV} / \mathrm{n}$ will be presented and discussed.

36th International Cosmic Ray Conference -ICRC2019-

July 24th - August 1st, 2019

Madison, WI, U.S.A.

\footnotetext{
* Speaker.

${ }^{\dagger}$ for collaboration list see PoS(ICRC2019)1177

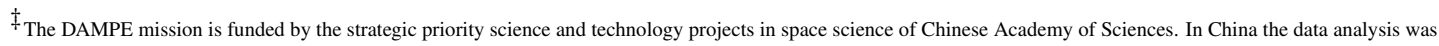
supported in part by the National Key Research and Development Program of China (No. 2016YFA0400200), the National Natural Science Foundation of China (Nos. 11525313, 11622327, 11722328, U1738133, U1738205, U1738207, U1738208), the strategic priority science and technology projects of Chinese Academy of Sciences (No. XDA15051100), the 100 Talents Program of Chinese Academy of Sciences, and the Young Elite Scientists Sponsorship Program. In Europe the activities and the data analysis are supported by the Swiss National Science Foundation (SNSF), Switzerland; the National Institute for Nuclear Physics (INFN), Italy.
} 


\section{Introduction}

Many different experiments are dedicated to the study of Cosmic Ray (CR) fluxes as a function of energy. In particular, great interest has been raised by the observation of special features in CR measured spectra, like some evident swift changes of the spectral index that break the single power-law trend of the flux. The presence of this kind of features indicates of something not yet understood in the propagation mechanisms, or in the acceleration processes or even in possible sources. One of these features is the so-called spectral hardening, already observed by several experiments [1]-[5] in the primary Helium nuclei (He) and Proton (p) fluxes at energies of hundreds of $\mathrm{GeV}$. Moreover, measurements at $\mathrm{TeV}$-energies $[2,5,6]$ show a spectral softening in $\mathrm{p}$ and $\mathrm{He}$ spectra, which motivate further investigations at higher energies. Preliminary results of the He-flux measurement performed by the DAMPE experiment will be here presented and discussed.

\section{DAMPE detector}

The DArk Matter Particle Explorer (DAMPE) [7, 8] is a detector hosted on-board a satellite, running on a Sun-Synchronous orbit with a period of $\sim 95$ minutes since December 17, 2015 at an altitude of $\sim 500 \mathrm{~km}$. On the top of it, a Plastic Scintillator Detector (PSD) [9], composed by two layers of plastic scintillator bars, measures the absolute value of the incoming CR charge up to $Z<28$ and provides an anti-coincidence veto signal for $\gamma$-rays. The bars in the first and in the second layer are parallel to the $\mathrm{x}$-axis and $\mathrm{y}$-axis of the DAMPE coordinate system ${ }^{1}$, respectively. The second sub-detector is the Silicon Tungsten tracKer-converter (STK) [10], made of 6 tracker planes used to reconstruct the trajectory, with the addition of 3 tungsten layers useful to boost the photon conversion in electron-positron pairs. Under the tracking-converter system, a calorimeter (BGO) [11], composed by 14 layers with $\mathrm{Bi}_{3} \mathrm{Ge}_{4} \mathrm{O}_{12}$ crystal bars, provides the measurement of the energy deposited by the crossing CR. Finally, at the bottom of the DAMPE detector, a NeUtron Detector (NUD) made of boron-doped plastic scintillator tiles allows to better discriminate between electromagnetic and hadronic showers.

\section{Data-taking and Helium candidate selection}

The analysis of the Helium spectrum here presented has been achieved by using 39 months of flight-data recorded from January 1st, 2016 to March 31th, 2019. They correspond to a detector livetime of $\sim 7.86 \cdot 10^{7} \mathrm{~s}$, by considering that $\sim 18.5 \%$ of the total time is instrumental dead time for the acquisition process, a $\sim 1.5 \%$ of the operation time is dedicated to the on-orbit calibration data-taking and finally, during the $\sim 4.5 \%$ of its working time, DAMPE crosses the South Atlantic Anomaly (SAA) region in which all the recorded events are excluded in the analysis.

\subsection{Pre-selection}

Before being analyzed, a recorded event must satisfy specific preliminary requisites. The first requirement is that the energy deposited by the incoming Cosmic Ray inside the whole BGO calorimeter has to be larger than $20 \mathrm{GeV}$, in order to avoid the influence of the geomagnetic cut-off

\footnotetext{
${ }^{1}$ The DAMPE coordinate system has been chosen in such a way that the z-axis is anti-oriented to the zenith, orthogonal to all the detector's planes and the y-axis points to the Sun.
} 

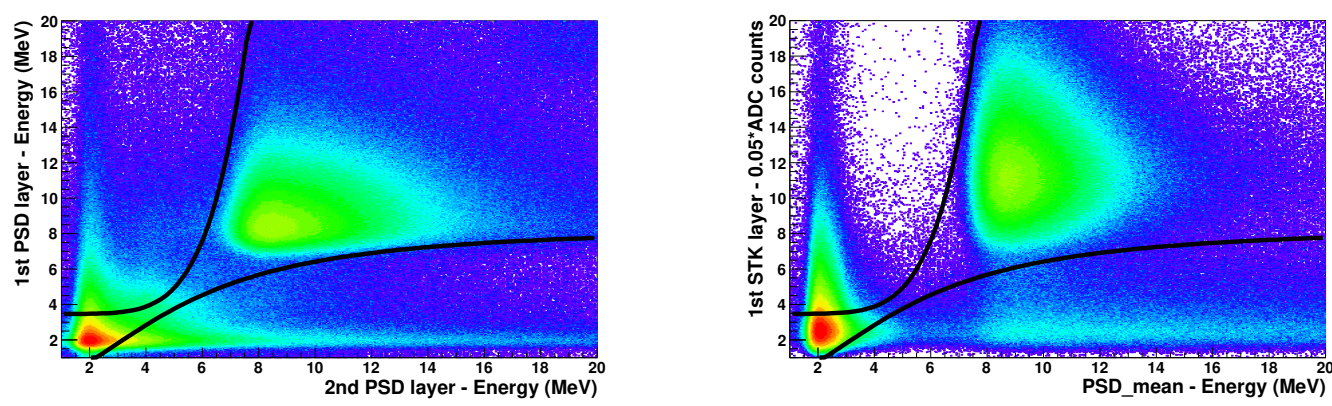

Figure 1: (Left) Energy correlation between the two PSD layers with 4 months of on-orbit data. The black lines indicate the PSD cut used to select the event sample. (Right) 1st STK layer signal as a function of mean energy deposited inside the two PSD layers, with 4 months of flight-data surviving the previous PSD selection cut. The black lines describe the subsequent cut applied.

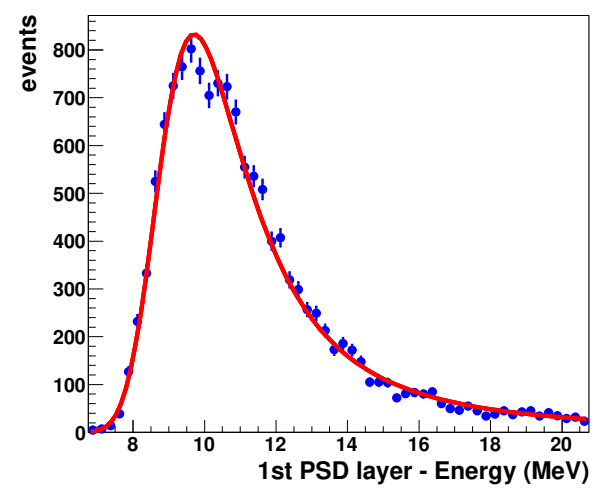

Figure 2: Energy distribution of the 1st PSD layer in the BGO energy range 1059.3 GeV-1496.2 GeV. Blue points represent flight-data and the red line is the convoluted Landau and Gaussian fitting functions.

effect. Several event selection criteria are then applied in order to find fully-contained events inside the BGO calorimeter. First of all, the maximum energy has not to be released in any of the edge bars of the first three BGO layers and the energy deposition in every layer has to be less than $35 \%$ of the total energy. This choice has been taken to exclude events generated by particles incident at large zenith angles. Furthermore, the reconstructed track inside the BGO has to be contained within the first and the last layers. Another requirement is the presence of at least one reconstructed track inside the STK. Moreover, this track has to match with the reconstructed direction of the shower inside the calorimeter and its projection has to cross within $20 \mathrm{~mm}$ the PSD bars with energy release greater than $0.5 \mathrm{MeV}$. These requirements constitute the so-called pre-selection.

\subsection{Event selection}

At this stage of the analysis, the selection of Helium candidates starts by asking as a first condition the activation of the High Energy Trigger (HET). This means an energy deposition greater than $\sim 10$ Minimum Ionizing Particles $\left(1 \mathrm{MIP}_{\mathrm{BGO}}=23 \mathrm{MeV}\right)$ in the first three layers of the BGO calorimeter and higher than $\sim 2$ MIPs inside the fourth BGO layer. The HET ensures a better reconstruction of the total energy deposition inside the calorimeter. The average HET rate is $\sim 50 \mathrm{~Hz}$, which implies a collection of more than $\sim 4 \mathrm{M}$ events every day with a total amount of $\sim 12 \mathrm{~GB} /$ day of data transferred to the ground. 


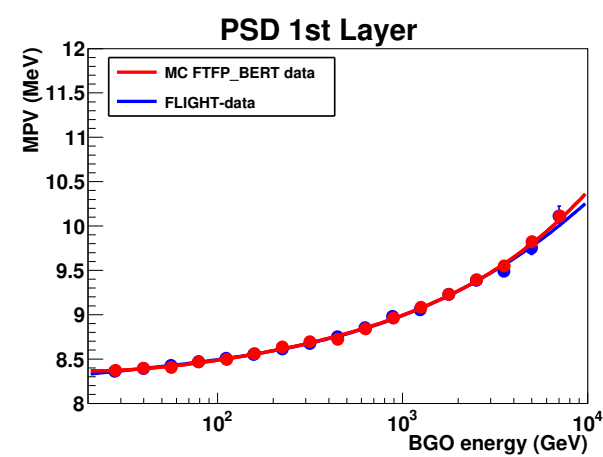

PSD 2nd Layer

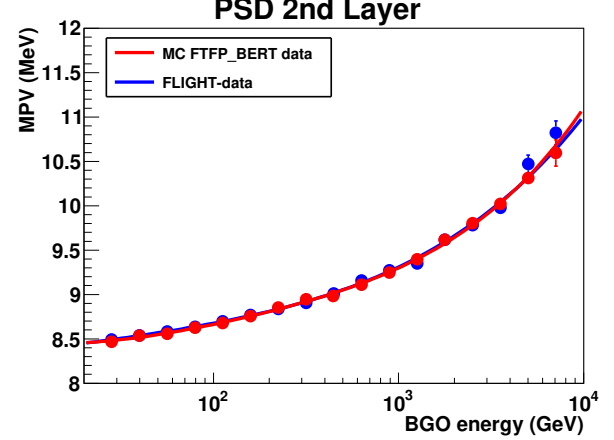

PSD 1st Layer

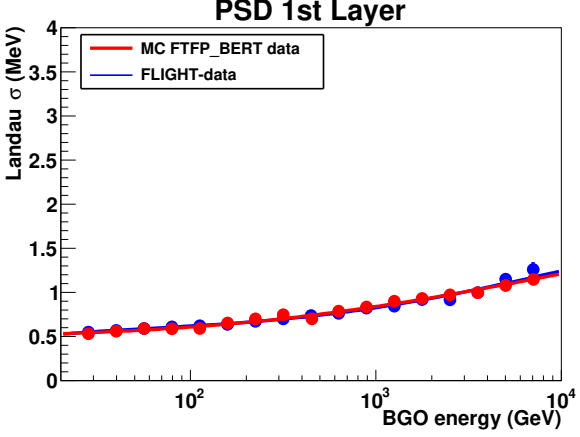

PSD 2nd Layer

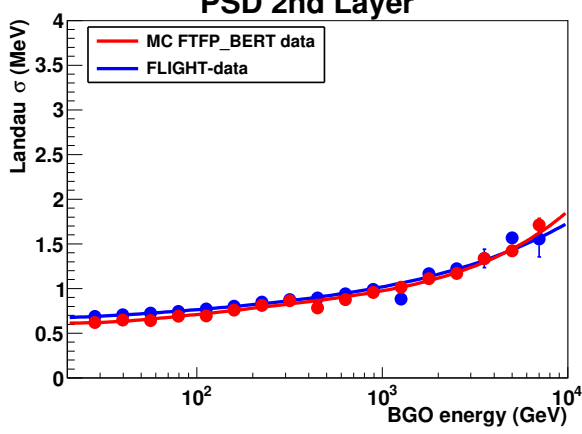

Figure 3: MPV and Landau $\sigma$ from the convoluted Landau and Gaussian fit of energy distributions inside the 1st and the 2nd PSD layers as a function of the energy deposition in the BGO calorimeter. Flight-data (blue points) have been compared with MC simulations (red points) for He nuclei in the energy range from $20 \mathrm{GeV}$ to $10 \mathrm{TeV}$.

Another requisite to select $\mathrm{He}$ candidates is that the energy deposition inside the first two layers of the BGO must be lower than the energy released in the third and fourth layers. Finally, two further analysis cuts have been applied to the surviving events. The first one consists in the selection of the sample by looking at the 1st PSD layer signal as a function of the 2nd layer signal, as shown in the left panel of Fig.1. The last event cut, plotted in the right panel of Fig.1, has been performed by looking at the ADC signal of the 1st STK layer as a function of the arithmetic mean energy in both the PSD layers. The contribution of these last two criteria, both tuned on the MC Helium sample, is particularly important for efficient suppression of Proton contamination in the Helium sample.

\subsection{Charge selection}

The incoming CR charge has been evaluated by considering the distributions of the energy loss measurements in the two views of the PSD, which are, according to the Bethe-Bloch formula, proportional to the square of the particle charge. By fitting these distributions for several BGO energy deposition ranges with a convolution of Landau and Gaussian functions, as in the example shown in Fig.2, the charge selection is finally performed by asking that the energy deposition inside each PSD layer has to be within [MPV $-2 \sigma$; MPV $+4 \sigma]$. The Most Probable Value (MPV) and the $\sigma$ are clearly dependent on the BGO energy deposition range in which they have been evaluated, as shown in Fig.3. The same procedure has been performed with MC simulation samples generated by using the GEANT4 toolkit [12] with the physics list FTFP_BERT for Helium nuclei with energies from $10 \mathrm{GeV}$ to $200 \mathrm{TeV}$ per nucleus. The comparison between flight-data and 

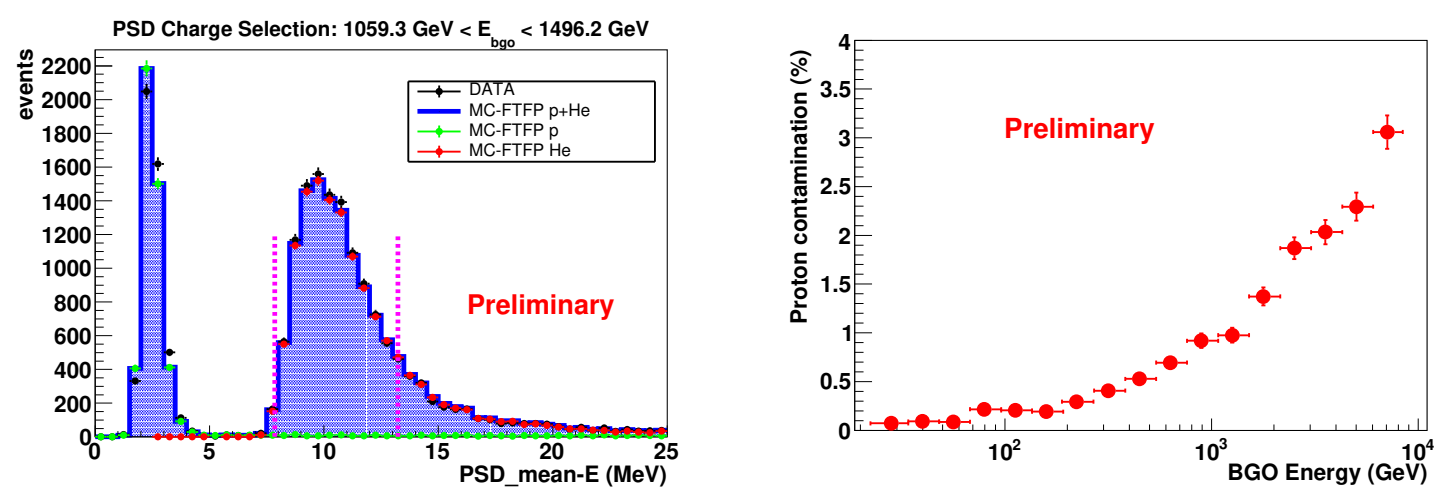

Figure 4: (Left) Distribution of the arithmetic mean energy deposited inside the two PSD layers in the BGO energy range between $1059.3 \mathrm{GeV}$ and $1496.2 \mathrm{GeV}$. Black points are flight-data, compared with the template fits of Proton MC (green points), Helium MC (red points) and Proton+Helium (blue line). The range in which Helium candidates are selected is pointed out with vertical dashed magenta lines obtained by using the presented charge selection criteria. (Right) Proton background percentage as a function of the energy deposition inside the BGO calorimeter.

MC simulations is necessary to validate the analysis and its selection cuts, but also to compute the Proton contamination inside the sample of selected Helium candidates. A difference between MC samples and flight-data in the PSD energy distributions as a function of the BGO energy deposition, due to back-splash effects, has been observed. Hence, a correction on MC simulations has been applied in order to achieve the agreement with on-orbit data. The Proton pollution has been estimated using the TFractionFitter ROOT class [13], as in the example of the left panel in Fig.4, with a sample of Proton MC in the energy range from $10 \mathrm{GeV}$ up to $1 \mathrm{PeV}$. In particular, the simulations from $10 \mathrm{GeV}$ to $100 \mathrm{TeV}$ have been performed by using the FTFP_BERT physics list, while from $100 \mathrm{TeV}$ to $1 \mathrm{PeV}$ the DPMJET interaction model has been adopted. Between $10 \mathrm{GeV}$ and $10 \mathrm{TeV}$ of energy deposition inside the BGO calorimeter, the resulting pollution percentage ranges from $\sim 0.07 \%$ up to $\sim 3 \%$, as shown in the right panel of Fig.4.

\section{Efficiencies}

\subsection{High Energy Trigger Efficiency}

Besides the HET, other three different triggers have been implemented in DAMPE. The most inclusive is the Unbiased trigger (Unb), activated everytime the signals in the first two BGO layers are greater than $\sim 0.4 \mathrm{MIPs}(9.2 \mathrm{MeV})$ in each hit BGO bar. This trigger is pre-scaled by $1 / 512$ when the satellite is in the geographical latitude range $\left[-20^{\circ}, 20^{\circ}\right]$ and $1 / 2048$ at higher latitudes. In order to compute the HET efficiency, the Unbiased trigger has been used as follows:

$$
\varepsilon_{H E T}=\frac{\mathrm{N}_{\mathrm{HET} \mid \mathrm{Unb}}}{\mathrm{N}_{\mathrm{Unb}}}
$$

where $\mathrm{N}_{\mathrm{HET} \mid \text { Unb }}$ and $\mathrm{N}_{\text {Unb }}$ are the numbers of Helium candidate events satisfying both the High Energy and Unbiased trigger requirements and the Unbiased activation only, respectively. The left panel of Fig.5 shows the HET efficiency as a function of the BGO energy deposition up to $10 \mathrm{TeV}$, computed both for on-orbit data and MC Helium candidate samples. The resulting difference 
between flight-data and MC simulations is within $\sim 5 \%$. Furthermore, the error bars of on-orbit data become larger at higher energies due to the pre-scaling of the Unbiased Trigger.
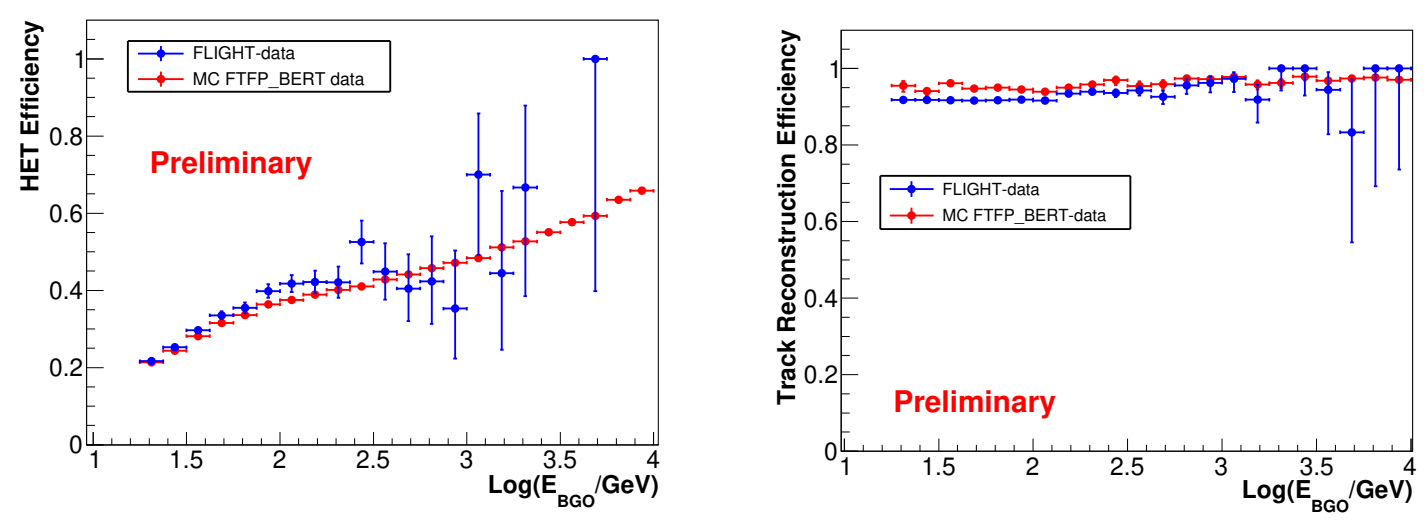

Figure 5: (Left) High Energy Trigger efficiency as a function of the energy deposited inside the BGO calorimeter for the selected Helium samples. The difference between flight-data (blue points) and MC simulations (red points) is found to be within $5 \%$ up to $10 \mathrm{TeV}$. (Right) STK track reconstruction efficiency as a function of the BGO energy deposition. Flight-data (blue points) and MC simulations (red points) are consistent within $3 \%$ up to $10 \mathrm{TeV}$.
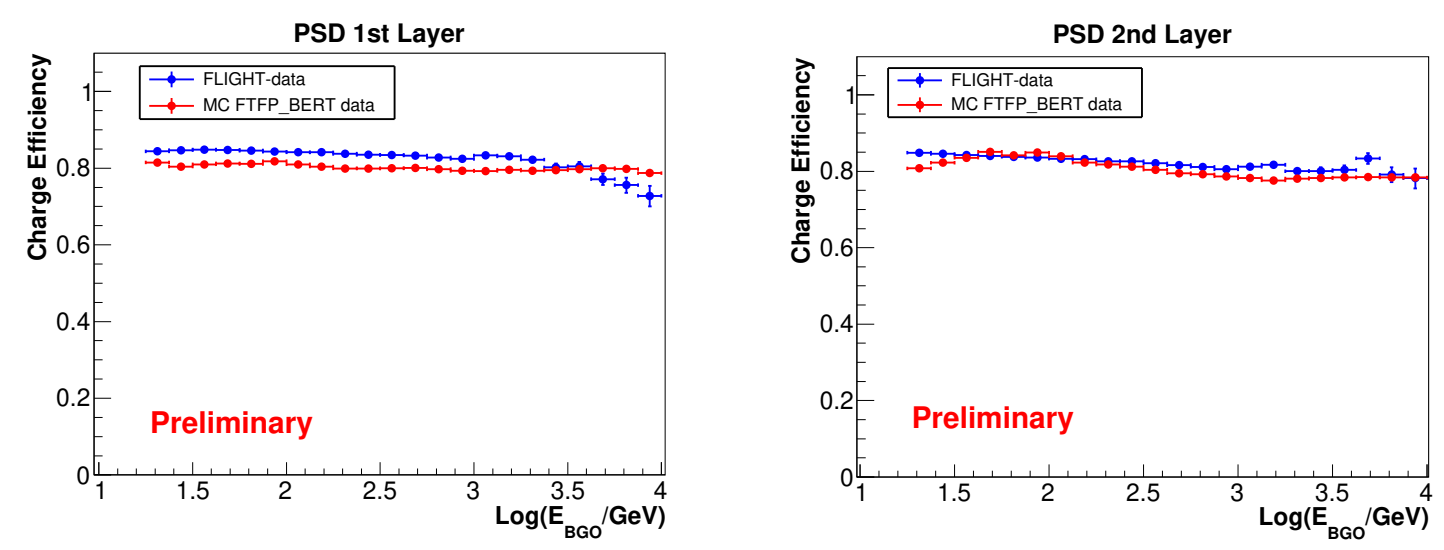

Figure 6: (Left) Charge reconstruction efficiency for the first layer of the PSD as a function of the energy deposited inside the BGO calorimeter. The agreement between flight-data (blue points) and MC simulations (red points) is within $4 \%$ up to $10 \mathrm{TeV}$. (Right) Charge efficiency for the second layer of the PSD. The difference between flight-data (blue points) and MC simulations (red points) is within $1 \%$ up to $10 \mathrm{TeV}$.

\subsection{Track Reconstruction Efficiency}

In order to estimate the STK track reconstruction efficiency, a comparison has been made between the Helium sample resulting from the standard analysis and the one selected by considering the reconstructed shower direction inside the BGO calorimeter which matches with the hit bars inside the PSD. The efficiency has been computed as follows:

$$
\varepsilon_{\text {Track }}=\frac{\mathrm{N}_{\mathrm{PSD}|\mathrm{STK}| \mathrm{BGO}}}{\mathrm{N}_{\mathrm{PSD} \mid \mathrm{BGO}}}
$$

where $\mathrm{N}_{\mathrm{PSD}|\mathrm{STK}| \mathrm{BGO}}$ is the number of events selected with the standard analysis criteria and $\mathrm{N}_{\mathrm{PSD} \mid \mathrm{BGO}}$ refers to the number of Helium candidate events selected by using the track informations provided 
by the PSD and the BGO. The right panel of Fig.5 shows the track reconstruction efficiency trend as a function of the BGO energy deposition, both for on-orbit data and MC simulations. The comparison shows a small difference between flight-data and MC within 3\% up to $10 \mathrm{TeV}$.

\subsection{Charge Reconstruction Efficiency}

The Charge reconstruction efficiency has been computed for both the PSD layers separately, with the help of the first STK layer charge measurement. For the 1st PSD layer, the following formula has been used:

$$
\varepsilon_{P S D 1 s t}=\frac{\mathrm{N}_{\text {PSD1st|PSD2nd|STK1st }}}{\mathrm{N}_{\text {PSD2nd|STK1st }}}
$$

which means a comparison between the Helium sample selected by using all the informations provided by the two PSD layers and the STK with the charge selection performed by using only the 2nd layer of the PSD combined with the STK. The same method has been used to estimate the 2nd PSD layer charge selection efficiency, by considering the samples selected with the 1st PSD layer together with the first layer of the STK. Fig.6 shows the charge reconstruction efficiencies for the 1st (left panel) and the 2nd (right panel) layer of the PSD. The consistency between flightdata and MC simulations is $\sim 4 \%$ for the first (outermost) and $\sim 1 \%$ for the second (innermost) layer, up to $10 \mathrm{TeV}$. The total systematic uncertainty for the charge reconstruction, computed as the quadrature sum of the two layers contributions, is $\sim 4.1 \%$.

\section{Preliminary results}

The effective acceptance for Helium nuclei selection has been computed starting from the geometrical factor $\mathrm{A}_{\text {gen }}$, used in the MC simulation of an isotropic flux of Cosmic Ray Helium nuclei generated above a sphere with a radius of $1.0 \mathrm{~m}$ and containing the DAMPE detector. Then, the effective acceptance at a given $i-$ bin of primary energy is given by:

$$
\mathrm{A}_{\text {eff }, i}=\mathrm{A}_{\text {gen }} \times \frac{\mathrm{N}_{\text {pass }, i}}{\mathrm{~N}_{\text {gen }, i}}
$$

where $\mathrm{N}_{\text {pass, } i}$ is the number of Helium candidate events which satisfied all the requirements of the previously discussed analysis, while $\mathrm{N}_{\mathrm{gen}, i}$ is the number of the total generated events, in the same $i$-bin of primary energy. The left panel of Fig.7 shows the effective acceptance as a function of the primary energy after all the selection cuts applied to the event sample, up to $\sim 20 \mathrm{TeV}$. The right panel in Fig.7 shows the preliminary $\mathrm{E}^{2.7}$ weighted Helium flux as a function of the primary energy per nucleon measured by DAMPE, compared with previous results of other experiments in Space. A spectral hardening is clearly observed at energies of few hundreds of $\mathrm{GeV}$ per nucleon, which suggests some hints for a better understanding of CR origin, propagation and acceleration mechanisms. Due to the limited thickness of the calorimeter ( $\sim 1.6$ interaction lenghts) only a part of the total energy is deposited inside the detector $(\sim 40 \%$ at $10 \mathrm{TeV})$. Hence, an unfolding procedure [14]-[16] has been adopted in order to reconstruct the primary energy of Helium nuclei. The grey band in the right panel of Fig.7 describes the total systematic uncertainty of the flux, computed in the following way:

$$
\sigma_{\text {sys }}=\sqrt{\sigma_{\mathrm{HET}}^{2}+\sigma_{\text {Track }}^{2}+\sigma_{\text {Charge }}^{2}+\sigma_{\text {Contamination }}^{2}} \simeq 7.2 \%
$$

Further studies on the systematic uncertainties due to the unfolding procedure and to the hadronic model are in progress. 

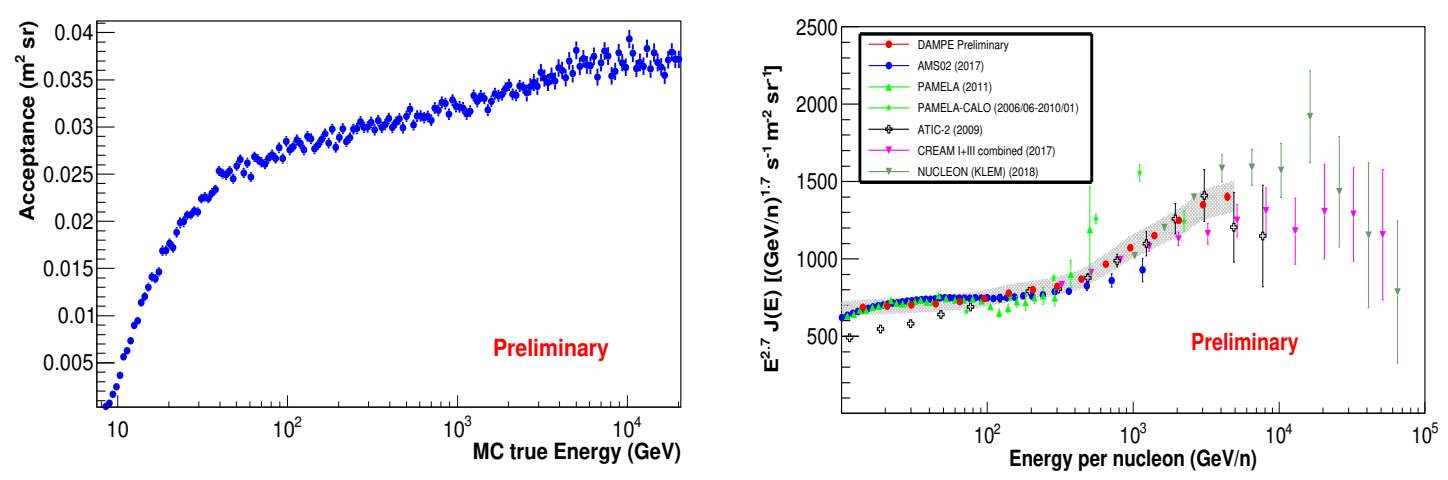

Figure 7: (Left) Effective acceptance for the Helium MC sample which satisfied every analysis selection requirements. (Right) Preliminary Helium flux weighted with $\mathrm{E}^{2.7}$ as a function of the primary energy per nucleon, compared with previous measurements performed by AMS-02 [1], CREAM I+III combined [2], PAMELA [3], PAMELA-CALO [4], ATIC-2 [5] and NUCLEON (KLEM) [6]. The dashed grey area describes the systematic uncertainties.

\section{Conclusions and outlooks}

Preliminary Helium spectrum has been measured with the DAMPE detector up to $\sim 5 \mathrm{TeV} / \mathrm{n}$, showing the spectral hardening evidence at energies of hundreds of GeV. The estimation of uncertainties is still on-going and the planned next goal is to reach energy of $100 \mathrm{TeV}$ for the Helium flux measurement in order to significantly improve our knowledge about the origin, acceleration and propagation mechanisms of Cosmic Rays.

\section{References}

[1] M. Anguilar et al. (AMS Collaboration), Phys. Rev. Lett. 119 (2017) 251101

[2] Y.S. Yoon et al. (CREAM Collaboration), Astrophys.J. 839 (2017) 1

[3] O. Adriani et al. (PAMELA Collaboration), Science 332 (2011) 69

[4] O. Adriani et al. (PAMELA Collaboration), AdSpR 51, 219 (2013)

[5] A.D. Panov et al. (ATIC Collaboration), Bull. Russ. Acad. Sci.: Phys. 73 (2009) 564

[6] E. Atkin et al. (NUCLEON Collaboration), JETP Lett. 108 (2018) 5-12

[7] J. Chang et al. (DAMPE Collaboration), Astropart. Phys. 95 (2017) 6-24

[8] G. Ambrosi et al., Astropart. Phys. 106 (2019) 18-34.

[9] Y. H. Yu et al., Astropart. Phys. 94 (2017) 1-10

[10] P. Azzarello et al., Nucl. Instrum. Meth. A 831 (2016) 378-384

[11] Z. Zhang et al., Nucl. Instrum. Meth. A 836 (2016) 98-104

[12] S. Agostinelli et al., Nucl. Instrum. Meth. A 506 (2017) 3

[13] https://root.cern.ch/doc/v606/classTFractionFitter.html

[14] G. D’Agostini, Nucl.Instrum.Meth. A 362 (1995) 487-498

[15] M.N. Mazziotta, arXiv:0912.1236 (2009)

[16] F. Loparco, M.N. Mazziotta, arXiv:0912.3695 (2009) 